\title{
Biomechanical Modeling of Human Body Movement
}

Tao Xiao ${ }^{1}$ and Yun-Feng $\mathrm{Fu}^{2^{*}}$

${ }^{1}$ Department of Physical Education, ZhengZhou University, PR China

${ }^{2}$ The Third Xiang-Ya Hospital, Central South University, Changsha, PR China

"Corresponding author: Yun-Feng Fu, The Third Xiang-Ya Hospital, Central South University, No.138 Tongzipo Road, Hexi District, Changsha, PR China, Tel: (86)07305320762; Fax: (86)07305320762; E-mail: yduy7927@163.com

Rec date: June 14, 2016; Acc date: June 23, 2016; Pub date: June 30, 2016

Copyright: ( 2016 Xiao T, et al. This is an open-access article distributed under the terms of the Creative Commons Attribution License, which permits unrestricted use, distribution, and reproduction in any medium, provided the original author and source are credited.

\begin{abstract}
The capabilities of the human body motion seem endless, through the long evolutionary process. The progresses made from the first step of a baby to an Olympic performance suggest that human movements have attained perfection in their specialized functions. However, the ability to predict how the whole body will move and how it will exchange forces with environment is becoming very vital for performances optimization or development of devices or safety; particularly in the fields of research of sport sciences, ergonomics, safety, clinical sciences and industries. Modeling human body motion is a huge issue due to the requirement of multifaceted researches obviously extremely diverse to apply. Indeed, they require the understanding of internal/external biological and physical principles that make possible and guide human movement and coordination, as well as, the capacity of giving them a realistic representation with high-fidelity.
\end{abstract}

Since over 30 years of research Biomechanics, the research area studying human motion has undertaken progress in the modeling human motion. But the results are mitigated. The purpose of this review is to report the state of knowledge and progress of the biomechanics regarding its application to the field of sport.

Keywords: Biomechanics; Human body; Movement; Sport optimization performances

\section{Introduction}

Human systems have great capabilities, highly specialized through long evolutionary system. The study of human locomotion for evaluating joint forces and moments that control motion and posture $[1,2]$ has been the fundamental to understand the mechanics of normal and pathological movement. It is an interdisciplinary task that describes, analyzes, and study human movement $[2,3]$ for the diagnosis and treatment of patients with motor deficiencies [3], for building intelligent devices which can be utilized for industrial, scientific and medical purposes.

Biomechanics, the field of science that uses laws of physics and engineering concepts to describe motion undergone by various body segments and the forces acting on these body parts during normal daily activities [4] has widely contributed in the progress of currents knowledge on human locomotion. Particularly, biomechanics of the human body has deal with body movements, with the forces that some parts of the human body exchange internally or externally, and with the effects that these movements and forces have on organs, and on the tissues that form them. Accordingly, the domains of interest of biomechanics are very huge, ranging across various great fields including:

- Physiology with a special focus on the musculoskeletal, cardiovascular, respiratory and digestive apparatuses;

- Pathology, specialised on orthopedics and traumatology, maxillofacial surgery, dentistry and orthodontist, cardiovascular and respiratory surgery;
- Forensics, specialised on accident reconstruction, crime scene investigation;

- Ergonomics and workplace safety, defense and social security, specialised on combat and law enforcement protection, effectiveness of projectile weapon; and

- Sport which is specialised on performance optimization, protection device.

Therefore, the realistic representation of biomechanical modeling of human movement is an issue which require the characterization of individual units of movements and incorporate them in sets of global movements and take in account the physiologic component involved. But, despite impressive evolution of Biomechanics, progresses made for modeling human motion are not great as one may have expected since over 30 years of research. Indeed, major questions still open. For examples, is it possible to know how to optimise movement in order to minimise the loads on the joints? How to better understand mechanics of injury and thus improve prevention? Other question related to the medical or technical equipment like- how to design the equipment to optimally suit the patient's or athlete's requirements in terms of mechanical behavioural? Or question related to body protection per se, as is the case for better design a cyclist helmets. Herein, we review the state of progress of the biomechanics of modeling of human body movement, regarding to application on sport.

\section{Fundamental issues of human motion control}

The motor unit and basic human body movements: The smallest subunit that can be controlled is called a motor unit [5] because it innervate separately by a motor axon. Neurologically, the motor unit consists of a synaptic junction in the ventral root of the spinal cord, a motor axon, and a motor end plate in the muscle fibers. Normal 
motion planning is initiated in the central nervous system (CNS). The sensorimotor control system in the brain generates a sequence of neural activation that innervates the muscle causing them to contract and generate the force required to drive a skeletal system to a desired position. Then sensory information such as muscle length, and skeleton motion parameters are fed back to the sensorimotor control skeletal muscle.

The bipedal movement: The difficulties associated with simple standing in upright humans are highlighted by increased risk of falling seen in the elderly, even with minimal reductions in control system effectiveness [6]. Bipedal movement requires many mechanical and neurological adaptations. The efficient energy means of standing in bipedal position involve constant adjustment of balance without overcorrection [6]. One of the illustrations can be demonstrated in infants who have not yet developed toward the ability to stand up. They can nevertheless run with great dexterity, provided they are supported in a vertical position and offered the stimulus of a moving treadmill beneath their feet [7]. Accordingly, walking is characterized by what researchers termed as an "inverted pendulum [8]" movement, in which the body vault over a stiff leg with each step. The model of inverted pendulum was applicable to all walking organisms regardless of the number of legs as the bipedal locomotion does not differ in term of whole body kinetics [9]. This contrast with running [10] which is characterized by a spring-mass movement, whereby the kinetic and potential energy are in phase, and energy is stored and released from spring-like limb during foot contact [11,12]. As well, all these movements required strong leg muscles and a functioning aerobic system [13]. Therefore, the realistic anatomical modeling for achieving high-fidelity of human animation was the major challenge to face in computer graphics, requiring many algorithms models that have been developed thus far [14-18].

\section{Modeling of Basic Human Body Movements}

From the difficulty to construct bipedal robot and/or their locomotion which involved only wheels, treads, or multiple legs, recent progress has made two-legged robot more feasible. Some notable biped robots have been created such as ASIMO [19], HUBO [20] and QRIO [21]. Accordingly significant progress focusing attention on biomechanically modeling of various parts of the human body have been developed, including the face [22], the neck [23], the torso $[14,24]$, the hand $[25,26]$ and the leg $[27,28]$. However, due to the complexity of the task, researchers have thus far shield away from undertaking a detailed biomechanical modeling of the entire human body. For example, the spine and torso which are particularly difficult to modeling have been drastically simplified in prior works. Either they were modeled in a strictly kinematic manner [29], grouping and treating many articular vertebrae and ribs as compound rigid bodies even in the most detailed dynamic models [24,30]. Relevant information in the report typically included the time histories of biomedical variables such as joint angle (kinematics) and joint moment (kinetics) [31] have been, however, taken in account for the development of custom applications. These information was particularly developed for feedback training using specific variable computed in real-time, such as a single angle [32] or a single joint moment [33]. There were approximations neglecting certain mechanical effects, such as inertial terms in the equation of motion [33], often introduced to make feasible the real-time computation. Accordingly, many real-time commercial systems are limited to kinematic variable (joint angles) [32,34] and possibly joint moments, but without muscle variable which is relevant for orthopedic or neurological rehabilitation or for computationally the whole body [35-37].

Recently, musculoskeletal models have been introduced to provide additional information about muscle length changes [38] and muscle forces $[35,36,39,40]$ within the most of complexity of human musculoskeletal system, which is due to the head-neck-trunk complex, have been solved. These parts of the body have approximately 57 articular bones and many more muscle actuators. In addition, the ribs form closed kinematic loops, which introduces additional complexities for biomechanical stimulation. Nonetheless, the comprehensive biomechanical modeling and control of the human upper body is the most principled approach to stimulating the full range of motions and deformation that is able to produce, from pronounced motions such as flexing the arms and spine to more subtle motions such as respiration and laryngeal movements or cardiac rhythms and so one.

Interestingly, more recently new devices have been developed with detailed biomechanical model of the human upper body, comprising the head, neck, trunk and arms for use in physics-based computer animation [41]. This kind of musculoskeletal system device displayed a full complement of muscle actuators and coupled finite element stimulation of soft tissue deformations $[42,43]$ giving more realistic representations of human models.

\section{Biomechanics Application to Sport}

The complexity of geometry of anatomical structures often requires a very high spatial resolution of the model leading to possibly demand of computer power. Complex device have been yield with acquisition of data by identification of anatomical structures of interest in $3 \mathrm{D}$ dataset derived from medical data with Computed Tomography (CT) or Magnetic Resonance Imaging (MRI) [44]. MRI advantage is to visualise muscles and ligaments in addition to the bony structure whereas CT gives a better representation of the bony structure. Both methods creating two-dimensional images with a predefined cilice thickness and slice distance of specified region. Devices description joint kinematics and the individual ability of those models influence human movement analysis, as reported for volleyball spikes [45] or a long jump and standing backwards somersault which allows the identification of the internal forces acting in the joints of biomechanical model of the human body [46].

Other biomechanical modeling developed analysis dealing with inverse problems, in which the important issue was the transfer of a real motion to the model and to find an optimal description of movement [47]. Some researchers have used reflective markers attach to the surrounding soft tissue of the shank and thigh to document general movement patterns, pathologies or sport related motions [42]. More comprehensive and complex biomechanical human models were also developed. One of the tremendous was developed for swimming athletes, with the stimulation framework implements the natural dynamic couplings between the flesh and skeleton, as well as between the deformable skin surface of the virtual human and the surrounding water, in an interleaved manner [48].

However, some care must be taken with all these sophisticated devices when interpreting results, because of the sensitivity regarding the procedure of smoothing the kinematic data. Indeed, it is important to collect a large number of video frames, particularly during the sporting and landing phase of the motion and the information provided by the measurements is either input for a model or used to 
Page 3 of 4

validate the model, such as comparing the simulation results with electromyography (EMG) data. In fact, the field of biomechanics suffers, unfortunately, from one very severe restriction which limits its full evolution. In general, it is not possible, for ethical reason, to measure forces and pressure inside the human body. The typical measurement technology in biomechanics works on the interface between body and environment. Force platforms dynamically quantify reaction forces when a person is walking or running across the sensor, EMG monitors action potentials of contracting muscles with electrodes attaches human skin. The information provided by measurement technologies are very important to investigate the mechanics of movements, but are not sufficient to answer some questions related to human body movement. As example, it is still not possible to know how to optimize movement in order to minimize the loads on the joints. As well, it is not possible to better understand mechanics of injury and thus improve prevention or to design the equipment's for optimally suit the patient's or athlete's requirements. Accordingly, the interior of an automobile is still always evaluated with rigid robot [49] for the impact of speed during accident or computer-aided engineering analysis device introduced to speed up the design of new high-comfort car seats. Thus, although the new devices are as realistic as possible, their conception is still estimating the real representation of muscle forces [50] with data which are not completely reflects of the reality.

\section{Conclusion}

Despite the impressive evolutions and the success of modeling techniques, progresses made in the field of biomechanics modeling seem emerged two major directions of development: the multi body system that have yield important results in prevention and sport [51-53] and the numerical models using finite elements or computational fluid dynamics, successfully applied to a variety of biomechanical problems such as improving injury prevention [54], ameliorating the design of equipment [55] and for optimizing movement techniques [56]. Each of the two models has his advantages and disadvantages. Globally, numerical models enable the computation of the whole body's deformation, whereas multi-body models only provide forces at a limited number of body points [57]. Thus the increasing availability of computational resources will lead to more complex models of the human body.

\section{References}

1. Fung Y (1990) Biomechanical aspects of growth and tissue engineering. Biomechanics.

2. Andersson GB, Winters JM (1990) Role of muscle in postural tasks: spinal loading and postural stability. Multiple muscle systems: Springerp.

3. Popovic D, Popovic M, Winters JM (2000) Nonanalytical control for assisting reaching in humans with disabilities. Biomechanics and Neural Control of Posture and Movement.

4. Nordin M, Frankel VH (2001) Basic biomechanics of the musculoskeletal system. Lippincott Williams \& Wilkins.

5. Thomas CK, Bakels R, Klein CS, Zijdewind I (2014) Human spinal cord injury: motor unit properties and behaviour. Acta Physiol (Oxf) 210: 5-19.

6. Chagdes JR, Rietdyk S, Jeffrey MH, Howard NZ, Raman A (2013) Dynamic stability of a human standing on a balance board. J Biomech 46: 2593-2602.

7. Berger W, Altenmueller E, Dietz V (1984) Normal and impaired development of children's gait. Hum Neurobiol 3: 163-170.

8. Qiao M, Jindrich DL (2014) Compensations during Unsteady Locomotion. Integr Comp Biol 54: 1109-1121.
9. Johnston IA (1984) Muscles and movement: muscles, reflexes, and locomotion. Science 226: 1308.

10. Cavagna GA, Kaneko M (1977) Mechanical work and efficiency in level walking and running. J Physiol 268: 467-467-81.

11. Segers V, De Smet K, Van Caekenberghe I, Aerts P, De Clercq D (2013) Biomechanics of spontaneous overground walk-to-run transition. J Exp Biol 216: 3047-3054.

12. Cavagna GA (1977) Storage and utilization of elastic energy in skeletal muscle. Exerc Sport Sci Rev 5: 89-129.

13. Marino FE (2008) The evolutionary basis of thermoregulation and exercise performance. Med Sport Sci 53: 1-13.

14. Zordan VB, Celly B, Chiu B, DiLorenzo PC (2006) Breathe easy: Model and control of human respiration for computer animation. Graphical Models 68: 113-132.

15. Wu G, Cavanagh PR (1995) ISB recommendations for standardization in the reporting of kinematic data. J Biomech 28: 1257-1261.

16. Conti G, Cristofolini L, Juszczyk M, Leardini A, Viceconti M (2008) Comparison of three standard anatomical reference frames for the tibiafibula complex. J Biomech 41: 3384-3389.

17. Lafortune MA, Cavanagh PR, Sommer HJ 3rd, Kalenak A (1992) Threedimensional kinematics of the human knee during walking. J Biomech 25: 347-357.

18. Ambrósio JA, Kecskeméthy A (2007) Multibody dynamics of biomechanical models for human motion via optimization. Multibody Dynamics.

19. Hirose M, Ogawa K (2007) Honda humanoid robots development. Philos Trans A Math Phys Eng Sci 365: 11-19.

20. Park I-W, Kim J-Y, Lee J, Oh J-H (2005) Mechanical design of humanoid robot platform KHR-3 (KAIST humanoid robot 3: HUBO). 5th IEEERAS International Conference on: IEEE.

21. Fujita M (2007) How to make an autonomous robot as a partner with humans: design approach versus emergent approach. Philosophical Transactions of the Royal Society of London A: Mathematical, Physical and Engineering Sciences 365: 21-47.

22. Stoiber N, Breton G, Seguier R (2010) Modeling short-term dynamics and variability for realistic interactive facial animation. IEEE Comput Graph Appl 30: 51-61.

23. Lee SH, Terzopoulos D (2006) Heads up!: biomechanical modeling and neuromuscular control of the neck. ACM Trans Graph 25: 1188-1198.

24. DiLorenzo PC, Zordan VB, Sanders BL (2008) Laughing out loud: control for modeling anatomically inspired laughter using audio. ACM Transactions on Graphics (TOG): ACM.

25. Albrecht I, Haber J, Seidel HP (2003) Construction and animation of anatomically based human hand models. pp: 98-109.

26. Sueda S, Kaufman A, Pai DK (2008) Musculotendon simulation for hand animation.

27. Komura T, Shinagawa Y, Kunii TL (2000) Creating and retargeting motion by the musculoskeletal human body model. The Visual Computer 16: 254-270.

28. Dong F, Clapworthy GJ, Krokos M, Yao J (2002) An anatomy-based approach to human muscle modeling and deformation. Visualization and Computer Graphics, IEEE Transactions on 8: 154-170.

29. Scheepers F, Parent RE, Carlson WE, May SF (1997) Anatomy-based modeling of the human musculature. ACM Press/Addison-Wesley Publishing Co.

30. Nakamura Y, Yamane K, Fujita Y, Suzuki I (2005) Somatosensory computation for man-machine interface from motion-capture data and musculoskeletal human model. Robotics IEEE Transactions 21: 58-66.

31. Kadaba MP, Ramakrishnan HK, Wootten ME, Gainey J, Gorton G, et al. (1989) Repeatability of kinematic, kinetic, and electromyographic data in normal adult gait. J Orthop Res 7: 849-860.

32. Barrios JA, Crossley KM, Davis IS (2010) Gait retraining to reduce the knee adduction moment through real-time visual feedback of dynamic knee alignment. J Biomech 43: 2208-2213. 
Page 4 of 4

33. Shull PB, Lurie KL, Cutkosky MR, Besier TF (2011) Training multiparameter gaits to reduce the knee adduction moment with data-driven models and haptic feedback. J Biomech 44: 1605-1609.

34. Teran-Yengle P, Birkhofer R, Weber MA, Patton K, Thatcher E, et al. (2011) Efficacy of gait training with real-time biofeedback in correcting knee hyperextension patterns in young women. J Orthop Sports Phys Ther 41: 948-952.

35. Delp SL, Anderson FC, Arnold AS, Loan P, Habib A, et al. (2007) OpenSim: open-source software to create and analyze dynamic simulations of movement. IEEE Trans Biomed Eng 54: 1940-1950.

36. Erdemir A, McLean S, Herzog W, van den Bogert AJ (2007) Model-based estimation of muscle forces exerted during movements. Clin Biomech (Bristol, Avon) 22: 131-154.

37. van den Bogert AJ, Geijtenbeek T, Even-Zohar O, Steenbrink F, Hardin EC (2013) A real-time system for biomechanical analysis of human movement and muscle function. Med Biol Eng Comput 51: 1069-1077.

38. Arnold AS, Liu MQ, Schwartz MH, Ounpuu S, Delp SL (2006) The role of estimating muscle-tendon lengths and velocities of the hamstrings in the evaluation and treatment of crouch gait. Gait Posture 23: 273-281

39. Heintz S, Gutierrez-Farewik EM (2007) Static optimization of muscle forces during gait in comparison to EMG-to-force processing approach. Gait Posture 26: 279-288.

40. van der Helm FC (1994) A finite element musculoskeletal model of the shoulder mechanism. J Biomech 27: 551-569.

41. Lee SH, Sifakis E, Terzopoulos D (2009) Comprehensive biomechanical modeling and simulation of the upper body. ACM Trans Graph 28: 1-17.

42. Benoit DL, Damsgaard M, Andersen MS (2015) Surface marker cluster translation, rotation, scaling and deformation: Their contribution to soft tissue artefact and impact on knee joint kinematics. J Biomech 48: 2124-2129.

43. Gao B, Zheng NN (2008) Investigation of soft tissue movement during level walking: translations and rotations of skin markers. J Biomech 41 : 3189-3195.

44. Eddy K, Ednie A, Connell C, Eddy R, Eaton K, et al. (2013) Appropriate use of CT and MRI in British Columbia. BCMJ.

45. Roemer K (2005) Influence of different knee models on calculated muscle forces.
46. Czaplicki A, Silva MT, Ambrósio JC (2004) Biomechanical modelling for whole body motion using natural coordinates. Journal of Theoretical and Applied Mechanics 42: 927-944.

47. Delp SL, Anderson FC, Arnold AS, Loan P, Habib A, et al. (1950) OpenSim: open-source software to create and analyze dynamic simulations of movement. Biomedical Engineering 54: 1940-1950.

48. Si W, Lee S-H, Sifakis E, Terzopoulos D (2014) Realistic Biomechanical Simulation and Control of Human Swimming. ACM Transactions on Graphics 34: 10-25.

49. Haddadin S, Albu-Schäffer A, Hirzinger G (2009) Requirements for safe robots: Measurements, analysis and new insights. The International Journal of Robotics Research 28: 1507-1527.

50. Grujicic M, Pandurangan B, Xie X, Gramopadhye A, Wagner D, et al. (2010) Musculoskeletal computational analysis of the influence of car-seat design/adjustments on long-distance driving fatigue. International Journal of Industrial Ergonomics 40: 345-355.

51. Gruber K, Ruder H, Denoth J, Schneider K (1998) A comparative study of impact dynamics: wobbling mass model versus rigid body models. J Biomech 31: 439-444.

52. Blajer W, Czaplicki A (2001) Modeling and inverse simulation of somersaults on the trampoline. J Biomech 34: 1619-1629.

53. Wojtyra M (2003) Multibody simulation model of human walking.

54. Brooks R, Mather J, Knowles S (2006) The influence of impact vibration modes and frequencies on cricket bat performance. Proceedings of the Institution of Mechanical Engineers. Part L: Journal of Materials Design and Applications 220: 237-248.

55. Dabnichki P, Avital E (2006) Influence of the postion of crew members on aerodynamics performance of two-man bobsleigh. J Biomech 39: 2733-2742.

56. Wangerin M, Schmitt S, Stapelfeldt B, Gollhofer A (2007) Inverse Dynamics in Cycling Performance. In. Advances in Medical Engineering: Springer pp: 329-334.

57. Penrose J, Hose D (1998) Finite element impact analysis of a flexible cricket bat for design optimisation. The Engineering of Sport-Design and Development. 